\title{
A constructive proof of Swap Local Search worst-case instances for the Maximum Coverage Problem
}

\author{
R.B.O. Kerkkamp ${ }^{\mathrm{a}, *}$, K. Aardal ${ }^{\mathrm{b}, \mathrm{c}}$ \\ a Econometric Institute, Erasmus University Rotterdam, P.O. Box 1738, 3000 DR Rotterdam, The Netherlands \\ ${ }^{\mathrm{b}}$ Delft Institute of Applied Mathematics, Delft University of Technology, Mekelweg 4, 2628 CD Delft, The Netherlands \\ ${ }^{\mathrm{c}}$ Centrum Wiskunde E Informatica (CWI), P.O. Box 94079, 1090 GB Amsterdam, The Netherlands
}

\section{A R T I C L E I N F O}

Article history:

Received 5 June 2015

Received in revised form

2 March 2016

Accepted 2 March 2016

Available online 10 March 2016

Keywords:

Maximum Weighted Coverage problem

Swap Local Search

Worst-case performance

Symmetry

Locality gap

\begin{abstract}
A B S T R A C T
We consider the Maximum Weighted Coverage problem (MCP). We can relate the MCP to optimisation problems using submodular functions. Performance guarantees of the Swap Local Search algorithm are known for these problems, but can be improved for the MCP. Our main contribution is a constructive proof of tight performance guarantees for Swap Local Search applied to the MCP, which provides insight into the structure of worst-case MCP instances, and has the potential to be applicable to other optimisation problems.
\end{abstract}

(C) 2016 Elsevier B.V. All rights reserved.

\section{Introduction}

For the Maximum Weighted Coverage problem (MCP) we are asked to open a limited number of 'bases', each with their own coverage, such that the combined coverage is maximised. The set $\ell$ denotes the set of all possible bases and up to $p \in \mathbb{N}$ bases can be selected. The coverage is defined using a set of 'demand points' $q$. Each opened base $i \in \ell$ covers a certain subset of demand points, indicated by the parameters $a_{i j} \in \mathbb{B}$ for $j \in$ g. That is, $a_{i j}=1$ if and only if base $i$ can cover point $j$. The demand parameter $d_{j} \in \mathbb{R}_{>0}$ is the objective coefficient of point $j \in \mathcal{G}$. Note that we assume non-negative demand weights.

The MCP is given by the following mathematical model:

maximise

$$
\sum_{j \in \mathcal{g}} d_{j} z_{j}
$$

subject to

$$
\begin{aligned}
\sum_{i \in \ell} x_{i} & \leq p, \\
\sum_{i \in \ell} a_{i j} x_{i} \geq z_{j} & \forall j \in \mathcal{g}, \\
x_{i}, z_{j} & \in \mathbb{B}
\end{aligned}
$$

\footnotetext{
* Corresponding author.

E-mail addresses: kerkkamp@ese.eur.nl (R.B.O. Kerkkamp), K.I.Aardal@tudelft.nl (K. Aardal).
}

The decision variable $x_{i}$ denotes whether we open (1) or close (0) base $i \in \ell$, and $z_{j}=1$ only if demand point $j \in \mathcal{g}$ is covered.

In the emergency medical service (EMS) literature this model is also known as the Maximal Covering Location problem (MCLP) [1], where the bases correspond to ambulances or ambulance bases. In other research fields demand points are called 'elements' in the universe $g$ and bases are sets of those elements. Both interpretations are equivalent and can be interchanged. Due to increased demand on punctuality and efficiency, EMS providers in many countries are using optimisation models to improve their performance. The MCP is the basis for many advanced EMS location models and is often used as a first assessment model.

The MCP is known to be NP-hard and Feige [2] proved that the best possible performance guarantee in polynomial time is $1-e^{-1}$ unless $P=N P$, see Theorem 1 .

Theorem 1 (Feige [2]). For any $\epsilon>0$ the MCP cannot be approximated in polynomial time with a guarantee of $1-e^{-1}+\epsilon$, unless $P=N P$.

Consequently, heuristics and approximation algorithms have been designed for the MCP. In particular, the simple Greedy Search attains the performance bound stated in Theorem 1, implying that it is the best possible approximation method in this sense (see [6]).

We are interested in performance guarantees for another typical heuristic: the Swap Local Search. Numerical results on both 
randomly generated and real-life MCP instances show that Swap Local Search reliably outperforms Greedy Search (see Section 4.5 in [5]). For the random instances, the Greedy Search has an average relative optimality gap of $2 \%$, and the Swap Local Search $1 \%$ (1Swap neighbourhood) or $0.2 \%$ (2-Swap neighbourhood). For the real-life instances, the relative improvement of the Swap Local Search with respect to the Greedy Search is of the same order.

Since the Greedy Search method has the best possible worstcase performance (unless $P=N P$ ), the Swap Local Search cannot always strictly outperform it. However, the Swap Local Search method could have the same worst-case performance. The numerical results indicate that the Swap Local Search is superior to Greedy Search, but what can be said about the worst-case?

\subsection{Swap Local Search performance guarantees}

To further discuss performance guarantees for the Swap Local Search, we have to specify the method in more detail. The Swap Local Search method iteratively improves an initial feasible solution by closing a subset of opened bases and opening a different subset of bases with strictly increased coverage. The two subsets are chosen such that they have the same cardinality and that the cardinality is at most $\rho \in \mathbb{N}_{>1}$. The resulting local maxima are called $\rho$-Swap local maxima. Since there are no costs associated with the bases in the MCP, it is optimal to open exactly $p$ bases. Therefore, we assume that we have an arbitrary initial feasible solution with $p$ opened bases. As a result, the total number of opened bases will not change by the Swap Local Search.

Let $\Omega$ be the set of all MCP instances. For each MCP instance $\omega \in \Omega$ we have a set of $\rho$-Swap local maximum solutions $\mathscr{L}(\omega)$ with objective values $\left\{\theta^{L}(\omega): L \in \mathcal{L}(\omega)\right\}$, and the global maximum $\theta^{*}(\omega)$. We can analyse the worst possible gap when considering all MCP instances and all corresponding local maxima. This tight bound is called the relative locality gap $\alpha \in[0,1]$ :

$\sup _{\omega \in \Omega, L \in \mathscr{L}(\omega)}\left\{\left(\theta^{*}(\omega)-\theta^{L}(\omega)\right) / \theta^{*}(\omega)\right\}=(1-\alpha)$.

The locality gap does not depend on which local maximum is actually reached by the used Swap Local Search algorithm. Therefore, the derived results are valid for any initial solution and any improvement method used by the algorithm. In this context, we call a performance bound tight if there exists a worst-case instance $\omega \in \Omega$ for which the bound is attained in a $\rho$-Swap local maximum $L \in \mathcal{L}(\omega)$.

Performance guarantees (bounds on the relative locality gap) for the Swap Local Search method are known for a broad class of optimisation problems related to submodular functions. A function $\phi: 2^{\mathcal{N}} \rightarrow \mathbb{R}$ for some finite discrete set $\mathcal{N}$ is called submodular if $\phi(\mathcal{U})+\phi(\mathcal{V})-\phi(\mathcal{V} \cap \mathcal{V}) \geq \phi(\mathcal{U} \cup \mathcal{V})$ for all $\mathcal{U}, \mathcal{V} \subseteq \mathcal{N}$. A submodular function has a decrease in marginal gain in function value in the sense that $\phi(\mathcal{U} \cup\{e\})-\phi(\mathcal{U}) \geq \phi(\mathcal{V} \cup\{e\})-\phi(\mathcal{V})$ for all $e \in \mathcal{N}$ and $U \subseteq \mathcal{V} \subseteq \mathcal{N} \backslash\{e\}$. Theorem 2 states performance guarantees for such optimisation problems.

Theorem 2 (Nemhauser, Wolsey, Fisher [6]). Let $\phi: 2^{\mathcal{N}} \rightarrow \mathbb{R}$ be a non-decreasing submodular function and $p \in \mathbb{N}$. Consider applying the $\rho$-Swap Local Search to the problem $\max \{\phi(\mathcal{U}):|u| \leq p$, $u \subseteq \mathcal{N}\}$ with an arbitrary initial feasible solution, where $p=a \bar{\rho}-b$ with $\rho, a \in \mathbb{N}_{\geq 1}$ and $b \in\{0, \ldots, \rho-1\}$. The following bound holds for any $\rho$-Swap local maximum $\theta^{L}$ and the global maximum $\theta^{*}$ :

$\frac{\theta^{*}-\theta^{L}}{\theta^{*}-\phi(\emptyset)} \leq \frac{p-\rho+b}{2 p-\rho+b}$.

If $b=0$, then this bound is tight.

As the MCP is a special case of the maximisation of a submodular function, the above result gives a bound on the locality gap for the MCP. Using the notation of Theorem 2, it holds for all MCP instances that $\left(\theta^{*}-\theta^{L}\right) / \theta^{*} \leq(p-\rho+b) /(2 p-\rho+b)$, since $\phi(\emptyset)=0$.

\subsection{Contribution}

Our main contribution is the technique used to prove tight bounds for the Swap Local Search method. This technique gradually builds an explicit description of the worst-case class of MCP instances. We use a sequence of instance transformations for which a 'worse' instance is obtained after each transformation. In particular, each transformation simplifies the structure and reduces the number of parameters of the instance. The procedure provides additional insight into the structure of 'worse' or worstcase MCP instances, namely that these instances have a certain symmetry as we will show.

This technique distinguishes itself from other approximation proofs, such as in [6,3], in the following way. Typical proofs first use optimality conditions and properties of the solution method to derive a performance bound. Instances do not play a role in this derivation. Then they find instances that reach the derived bound to show that the analysis is tight. Both steps together prove that these are worst-case instances. In contrast, our technique builds provably worst-case instances from the ground up. Here, we determine bounds and find worst-case instances simultaneously, not in two separate steps.

We show that with our technique the performance guarantee of Theorem 2 can be (slightly) improved for the MCP. We derive a tight bound based on the number of matching bases between the optimum and the $\rho$-Swap local maximum solution. In particular, the $\rho$-Swap Local Search method for the MCP has a locality gap of $\alpha=1 / 2$. Note that in terms of approximation ratios, this bound of $1 / 2$ for the Swap Local Search is known $[6,4]$. The tight performance guarantee for the Swap Local Search method is given in Theorem 3.

Theorem 3. Consider an arbitrary MCP instance $\omega \in \Omega$ with global maximum $\theta^{*}(\omega)$ and optimal solution $x^{*}(\omega)$. Let $x^{L}(\omega)$ be any $\rho$ Swap local optimum $\left(\rho \in \mathbb{N}_{\geq 1}\right)$ for $\omega$ with objective value $\theta^{L}(\omega)$.

Suppose $x^{*}(\omega)$ and $x^{L}(\omega)$ differ in exactly $2 k(\omega)$ elements for some $k(\omega) \in \mathbb{N}_{>\rho}$, that is, $\sum_{i \in \ell}\left|x_{i}^{*}(\omega)-x_{i}^{L}(\omega)\right|=2 k(\omega)$. Then we can bound the relative optimality gap by

$\frac{\theta^{*}(\omega)-\theta^{L}(\omega)}{\theta^{*}(\omega)} \leq \frac{k(\omega)-\rho}{2 k(\omega)-\rho}$,

and this bound is tight.

We place two remarks on the result of Theorem 3. First, when applying Theorem 3 we typically do not know the value of $k(\omega)$ and need to take $k(\omega)=p$ as a safe upper bound. As such, it provides only a slight improvement to Theorem 2 for the case $b \neq 0$. Second, in [6] the authors mention that they have established the bound $(p-\rho) /(2 p-\rho)$ for the maximisation version of the Uncapacitated Location problem. Unfortunately, no proof or further reference is given. Therefore, we could not compare our approach to theirs.

The rest of this paper consists of illustrating our technique by proving Theorem 3, which is split over several lemmas. For a more elaborate version of the proof, we refer to Section 4.4.2 of [5].

\section{The swap locality gap}

The proof of Theorem 3 is based on constructing a new MCP instance with the same or a larger relative optimality gap than the original instance, but with a simplified structure. This process of simplification is repeated until a family of (worst-case) instances remains for which the locality gap is trivial.

The simplification steps, and the resulting instance transformations, can be characterised as follows:

1. remove unnecessary bases and demand points,

2. create a bijection between bases and demand points,

3. use the inherent symmetry of the coverage problem,

4. simplify the structure of the instance. 
These instance transformations satisfy a specific set of properties. Let $\rho \in \mathbb{N}_{\geq 1}$ and $k \in \mathbb{N}$. Consider an arbitrary MCP instance $\omega \in \Omega$ where $x^{*}(\omega)$ and $x^{L}(\omega)$ differ in exactly $2 k$ elements ( $k$ swaps), see also the statement in Theorem 3. Note that $k \geq \rho+1$ must hold. The proof sequentially transforms $\omega$ into a new instance $\omega^{\prime}$ such that the following four properties are preserved:

1. $x^{*}(\omega)$ is an optimal solution for $\omega^{\prime}$,

2. $x^{L}(\omega)$ is a $\rho$-Swap local optimum for $\omega^{\prime}$,

3. $x^{L}(\omega)$ differs from $x^{*}(\omega)$ in exactly $2 k$ elements for $\omega^{\prime}$,

4. the corresponding optimality gap for $\omega^{\prime}$ is as least as large as that for $\omega$.

In the end, we obtain a family of worst-case instances for which we can derive the tight bound on the relative optimality gap.

\subsection{Step 1: clean-up}

The first transformation $\phi_{1}: \Omega \rightarrow \Omega$ removes redundant demand points and bases (a projection to a lower dimension). We transform the instance $\omega$ to a new instance where some of the common coverage between the global and $\rho$-Swap local maxima is eliminated.

Let $\ell^{*}(\omega)=\left\{i \in \ell(\omega): x_{i}^{*}(\omega)=1\right\}$ be the optimal set of opened bases and likewise $\ell^{L}(\omega)=\left\{i \in \ell(\omega): x_{i}^{L}(\omega)=1\right\}$. Construct a new MCP instance $\omega^{\prime}=\phi_{1}(\omega)$ where: the demand points not covered by any base in $\ell^{*}(\omega) \cup l^{L}(\omega)$ are removed; the demand points covered by any base in $\ell^{*}(\omega) \cap \ell^{L}(\omega)$ are removed; the bases in $\left\{i \in \ell(\omega): x_{i}^{*}(\omega)=x_{i}^{L}(\omega)\right\}$ are removed; and the number of opened bases is reduced to $p\left(\omega^{\prime}\right)=p(\omega)-\mid \ell^{*}(\omega) \cap$ $\ell^{L}(\omega) \mid$. No other changes are performed. See Fig. 1 for an example of this transformation. Bases are depicted as squares and demand points as black dots. The circles indicate the coverage of the bases.

The solution $x_{i}^{*}\left(\omega^{\prime}\right)=x_{i}^{*}(\omega)$ for $i \in \ell\left(\omega^{\prime}\right)$ is optimal for $\omega^{\prime}$ and $x_{i}^{L}\left(\omega^{\prime}\right)=x_{i}^{L}(\omega)$ for $i \in \ell\left(\omega^{\prime}\right)$ is a $\rho$-Swap local optimum. As in the original instance, $x^{*}\left(\omega^{\prime}\right)$ and $x^{L}\left(\omega^{\prime}\right)$ differ in exactly $2 k$ elements. Furthermore, the relative optimality gap of $\omega^{\prime}$ is as least as large as that of $\omega$. Hence, the four properties mentioned above still hold.

\subsection{Step 2: create a bijection}

Consider a new arbitrary MCP instance $\omega \in \phi_{1}(\Omega)$ where $x^{*}(\omega)$ and $x^{L}(\omega)$ differ in exactly $2 k$ elements. Note that $|\ell(\omega)|=2 k$, so the two solutions have no matching elements. For the remainder of the proof, we fix the set of bases, $\ell(\omega)=\ell$, as well as both solutions, $x^{*}(\omega)=x^{*}$ and $x^{L}(\omega)=x^{L}$.

Each demand point can be covered by a certain subset of bases in $\ell$. Thus, we can project each point to the largest subset of bases that can cover it, denoted by $\pi: \mathcal{g}(\omega) \rightarrow 2^{\ell}$. This projection is not guaranteed to be an injection nor a surjection.

The second instance transformation will merge certain demand points and add new artificial demand points. We can divide the demand points into several sets, where two points $j_{1}, j_{2} \in \mathcal{g}(\omega)$ are in the same set if and only if both are covered by the same bases: $\pi\left(j_{1}\right)=\pi\left(j_{2}\right)$. Construct a new MCP instance by merging the demand points in each of these sets. Note that $\pi$ is injective for this new instance. Next, add artificial demand points with zero demand to the new instance in such a way that $\pi$ becomes bijective. Call this transformation $\phi_{2}: \phi_{1}(\Omega) \rightarrow \phi_{1}(\Omega)$. It is trivial that $\phi_{2}$ does not affect feasibility or objective values, thus the four properties still hold.

We fix this set of demand points for the remainder of the proof, $g\left(\omega^{\prime}\right)=g$. As a result, the bijection $\pi$ for $\omega^{\prime}=\phi_{2}(\omega)$ has an inverse $\pi^{-1}: 2^{\ell} \rightarrow \mathcal{g}$, see also Lemma 4 and Fig. 2. For example, demand point $\pi^{-1}(\{i\})$ can only be covered by base $i \in \ell$, demand point $\pi^{-1}\left(\left\{i_{1}, i_{2}\right\}\right)$ can be covered by exactly two bases $\left(i_{1}\right.$ and $\left.i_{2}\right)$, etcetera. For clarity, we omit the bijection in demand values, i.e., $d_{\pi^{-1}\left(\left\{i_{1}, i_{2}\right\}\right)}(\omega)$ is abbreviated to $d_{\left(\left\{i_{1}, i_{2}\right\}\right)}(\omega)$ and similarly for the other subsets of bases.
Lemma 4. For each MCP instance $\omega \in \Omega$ there exists an MCP instance $\omega^{\prime} \in \Omega$ with the bijective mapping $\pi: g\left(\omega^{\prime}\right) \rightarrow 2^{\ell\left(\omega^{\prime}\right)}$, that maps each demand point to the largest subset of bases that can cover it.

For the new MCP instance $\omega^{\prime}, x^{*}(\omega)$ is still the global optimum and $x^{L}(\omega)$ is still a $\rho$-Swap local optimum, although a projection to a lower dimension can be required. Furthermore, the relative optimality gap of $\omega^{\prime}$ is as least as large as that of $\omega$.

Proof. The proof has been given above by taking $\omega^{\prime}=\phi_{2}\left(\phi_{1}\right.$ $(\omega))$.

The constructed bijection allows us to explicitly express our situation as linear constraints. Let $\omega \in \phi_{2}\left(\phi_{1}(\Omega)\right)$ be an arbitrary instance, where $x^{*}$ and $x^{L}$ differ in exactly $2 k$ elements. Define the disjoint sets $\ell^{*}=\left\{i \in \ell: x_{i}^{*}=1\right\}$ and $\ell^{L}=\left\{i \in \ell: x_{i}^{L}=1\right\}$. We can express the global and local maxima by

$$
\begin{aligned}
& \theta^{L}(\omega)=\sum_{\emptyset \neq y^{L} \subseteq l^{L}} \sum_{y^{*} \subseteq l^{*}} d_{\left(y^{L} \cup y^{*}\right)}(\omega), \\
& \theta^{*}(\omega)=\sum_{\emptyset \neq y^{*} \subseteq l^{*}} \sum_{y^{L} \subseteq l^{L}} d_{\left(y^{L} \cup y^{*}\right)}(\omega) .
\end{aligned}
$$

In the definition of $\theta^{L}(\omega)$, the first summation selects a non-empty subset $y^{L}$ of bases in $\ell^{L}$ and the last selects a possibly empty subset $y^{*}$ of $\ell^{*}$. The corresponding demand point is a point covered by at least one base in $\ell^{L}$. Thus, the local maximum is equal to all demand from the points that are covered by at least one base in $\ell^{L}$. Likewise, for the global maximum at least one base from $\ell^{*}$ is required.

For example, consider Fig. 2(b). Omitting demand terms equal to zero, we have the local maximum: $\theta^{L}=\left(d_{(\{A\})}+d_{(\{B\})}\right)+$ $\left(d_{(\{A, C\})}+d_{(\{B, C\})}+d_{(\{B, D\})}\right)+\left(d_{(\{B, C, D\})}\right)+\left(d_{(\{A, B\})}\right)+\left(d_{(\{A, B, C\})}\right)$. Here, we have grouped the terms according to the cardinalities of $y^{L}$ and $y^{*}$ as in Eq. (1).

What remains is the expression of the properties of the $\rho$-Swap local maximum and the global maximum. Since the global maximum is equivalent to a $k$-Swap local maximum, we can focus on expressing Swap local maxima as constraints. Consider the $\rho$-Swap local optimum $\left(x^{L}\right)$ and the swap where $i^{L} \in \ell^{L}$ is replaced by $i^{*} \in \ell^{*}$. The following demand is the net loss of this swap:

$\sum_{y^{*} \subseteq l^{*} \backslash\left\{i^{*}\right\}} d_{\left(\left\{i^{L}\right\} \cup y^{*}\right)}(\omega)$.

The expression is equal to the demand of all points covered by base $i^{L}$ and simultaneously covered by any base or multiple bases in $\ell^{*} \backslash\left\{i^{*}\right\}$. Similarly, the following demand is the net gain:

$\sum_{y^{*} \subseteq l^{*} \backslash\left\{i^{*}\right\}} d_{\left(\left\{i^{*}\right\} \cup y^{*}\right)}(\omega)$.

The net gain is equal to the demand of all points covered by $i^{*}$ and simultaneously covered by any base or multiple bases in $l^{*} \backslash\left\{i^{*}\right\}$. The net effect of each swap must be non-positive, since $x^{L}$ is in particular a 1-Swap local optimum. Thus, for all $i^{*} \in \ell^{*}$ and $i^{L} \in \ell^{L}$ :

$\sum_{y^{*} \subseteq l^{*} \backslash\left\{i^{*}\right\}}\left(d_{\left(\left\{i^{*}\right\} \cup y^{*}\right)}(\omega)-d_{\left(\{i L\} \cup y^{*}\right)}(\omega)\right) \leq 0$.

For example, in the situation of Fig. 2(b), we can swap base $B$ from $\ell^{L}$ with base $C$. The corresponding constraint is given by $\left(d_{(\{C\})}-d_{(\{B\})}\right)+\left(d_{(\{C, D\})}-d_{(\{B, D\})}\right) \leq 0$.

In general, consider swapping $R \in\{1, \ldots, \rho\}$ bases in $\ell^{L}$ with bases in $\ell^{*}$. We swap $U^{L} \subseteq \ell^{L}$ with $U^{*} \subseteq \ell^{*}$, both with cardinality $R$. The effect of the swap is as follows. We keep any demand covered by bases in $\ell^{L} \backslash \mathcal{U}^{L}$. We lose demand that is covered only by bases in $U^{L}$ and gain demand that will be covered only by bases in $U^{*}$. For the net effect, we have to take into account that the coverage 


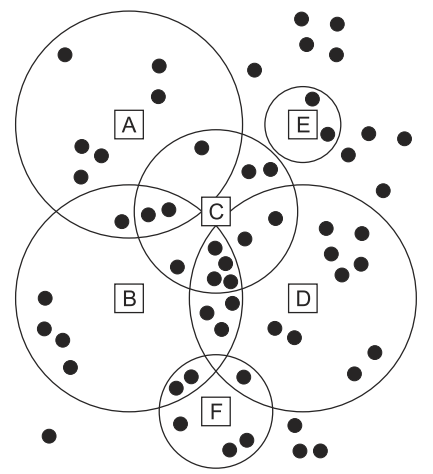

(a) Instance $\omega \in \Omega$.

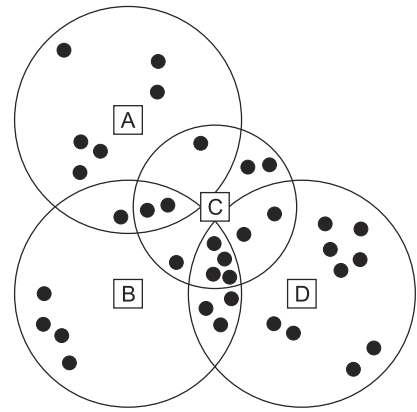

(b) New instance $\phi_{1}(\omega)$.

Fig. 1. First instance transformation $\phi_{1}$, assuming $\ell(\omega)=\{A, B, C, D, E, F\}, p(\omega)=3, k(\omega)=2, \ell^{L}(\omega)=\{A, B, F\}$, and $\ell^{*}(\omega)=\{C, D, F\}$.

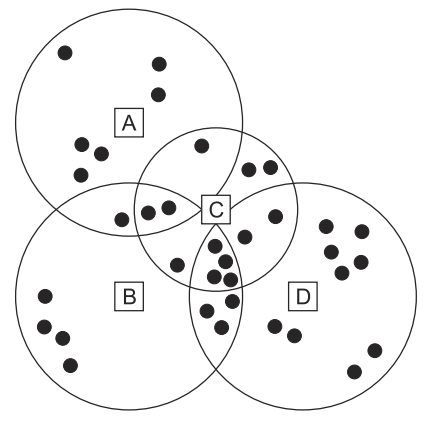

(a) Instance $\omega \in \phi_{1}(\Omega)$.

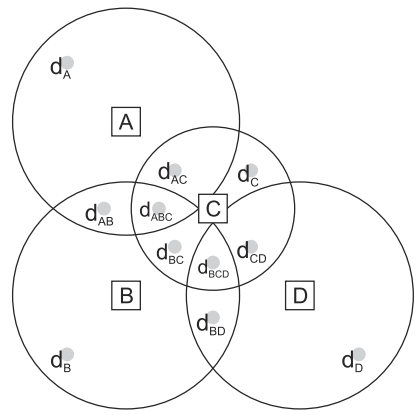

(b) New instance $\omega^{\prime}=\phi_{2}(\omega)$.

Fig. 2. Second instance transformation $\phi_{2}$, assuming $\ell(\omega)=\{A, B, C, D\}, p(\omega)=2, \ell^{L}(\omega)=\{A, B\}$, and $\ell^{*}(\omega)=\{C, D\}$. Artificial demand points are not shown.

of $U^{L}$ and $U^{*}$ can overlap. Thus, the net loss is

$\sum_{\emptyset \neq \mathcal{Z}^{L} \subseteq u^{L}} \sum_{y^{*} \subseteq l^{*} \backslash u^{*}} d_{\left(\mathcal{Z}^{L} \cup y^{*}\right)}(\omega)$

and the net gain is

$\sum_{\emptyset \neq \mathcal{Z}^{*} \subseteq u^{*}} \sum_{y^{*} \subseteq l^{*} \backslash u^{*}} d_{\left(Z^{*} \cup y^{*}\right)}(\omega)$.

The expressions simply keep track of the demand values using the bijection $\pi$. As before, the summations select subsets, where $\mathcal{Z}^{L} \subseteq \mathcal{U}^{L}$ and $\mathfrak{Z}^{*} \subseteq \mathcal{U}^{*}$ must be non-empty. Compare these expressions to Eq. (1).

Therefore, the following $\rho$-Swap optimality constraints must hold for all $R \in\{1, \ldots, \rho\}, U^{L} \subseteq \ell^{L}$ with $\left|U^{L}\right|=R$, and $U^{*} \subseteq \ell^{*}$ with $\left|U^{*}\right|=R$ :

$$
\begin{aligned}
& \sum_{\emptyset \neq \mathcal{Z}^{*} \subseteq u^{*}} \sum_{y^{*} \subseteq l^{*} \backslash u^{*}} d_{\left(Z^{*} \cup y^{*}\right)}(\omega) \\
& -\sum_{\emptyset \neq \mathcal{Z}^{L} \subseteq u^{L}} \sum_{y^{*} \subseteq l^{*} \backslash u^{*}} d_{\left(Z^{L} \cup y^{*}\right)}(\omega) \leq 0 .
\end{aligned}
$$

The constraints for the global optimum $\left(x^{*}\right)$ are similar, but the roles of $U^{L}$ and $U^{*}$ are interchanged. Therefore, the following constraints must hold for all $R \in\{1, \ldots, k\}, U^{L} \subseteq \ell^{L}$ with $\left|U^{L}\right|=$ $R$, and $U^{*} \subseteq \ell^{*}$ with $\left|U^{*}\right|=R$ :

$$
\begin{aligned}
& \sum_{\emptyset \neq \mathcal{Z}^{L} \subseteq u^{L}} \sum_{y^{L} \subseteq l^{L} \backslash u^{L}} d_{\left(\mathcal{Z}^{L} \cup y^{L}\right)}(\omega) \\
& -\sum_{\emptyset \neq \mathcal{Z}^{*} \subseteq u^{*}} \sum_{y^{L} \subseteq l^{L} \backslash u^{L}} d_{\left(\mathcal{Z}^{*} \cup y^{L}\right)}(\omega) \leq 0 .
\end{aligned}
$$

In the case of Fig. 2(b), the constraint when swapping $\ell^{*}$ with $\ell^{L}$ is: $\left(d_{(\{A\})}+d_{(\{B\})}+d_{(\{A, B\})}\right)-\left(d_{(\{C\})}+d_{(\{D\})}+d_{(\{C, D\})}\right) \leq 0$.

All derived linear constraints completely describe our situation with the global and Swap local optima.

\subsection{Step 3: use symmetry}

The third instance transformation constructs a more symmetric instance and will be defined below (see also Fig. 3). With this transformation we can prove the following lemma.

Lemma 5. Consider an MCP instance $\omega \in \phi_{2}\left(\phi_{1}(\Omega)\right)$ where the global optimum $x^{*}(\omega)$ and $\rho$-Swap local optimum $x^{L}(\omega)$ differ at $2 k$ elements. There is a modified MCP instance $\omega^{\prime} \in \phi_{2}\left(\phi_{1}(\Omega)\right)$ with the symmetric properties that for all $n, m \in\{0, \ldots, k\}, y^{L} \subseteq \ell^{L}\left(\omega^{\prime}\right)$ with $\left|y^{L}\right|=n$, and $y^{*} \subseteq \ell^{*}\left(\omega^{\prime}\right)$ with $\left|y^{*}\right|=m$ :

$d_{\left(y\left\llcorner\cup y^{*}\right)\right.}\left(\omega^{\prime}\right)=d_{(n, m)}\left(\omega^{\prime}\right)$.

For the new instance $\omega^{\prime}, x^{*}(\omega)$ is still the global optimum and $x^{L}(\omega)$ still a $\rho$-Swap local optimum. Furthermore, the relative optimality gap of $\omega^{\prime}$ is equal to that of $\omega$.

Proof. Let $\omega \in \phi_{2}\left(\phi_{1}(\Omega)\right)$ be an arbitrary instance where the global optimum and $\rho$-Swap local optimum differ at $2 k$ elements. Let $\sigma^{*}: \ell^{*} \rightarrow \ell^{*}$ be a permutation of the bases in $\ell^{*}$ and $\sigma^{L}: \ell^{L} \rightarrow \ell^{L}$ a similarly defined permutation. Note that $\sigma^{*}$ and $\sigma^{L}$ are disjoint. If we combine the two permutations there are $k ! k$ ! permuted instances $\sigma_{s}(\omega)$, with $s \in\{1, \ldots, k ! k !\}$. The solutions $x^{*}$ and $x^{L}$ are global and local optima for all permuted instances $\sigma_{s}(\omega)$, as only the labels of the bases are changed within each set $l^{*}$ and $l^{L}$.

Construct a new MCP instance $\omega^{\prime}$ by taking the average demand of the permuted instances in the following way. For $y^{L} \subseteq \ell^{L}$ and $y^{*} \subseteq \ell^{*}$ define

$$
\begin{aligned}
d_{\left(y^{L} \cup y^{*}\right)}\left(\omega^{\prime}\right) & =\frac{1}{k ! k !} \sum_{s=1}^{k ! k !} d_{\left(y^{L} \cup y^{*}\right)}\left(\sigma_{s}(\omega)\right) \\
& =\frac{1}{k ! k !} \sum_{s=1}^{k ! k !} d_{\left(\sigma_{s}\left(y^{L} \cup y^{*}\right)\right)}(\omega) .
\end{aligned}
$$


As all constraints (2) and (3) are linear in $d$ and valid for each $\sigma_{s}(\omega)$, these are also valid for the new instance $\omega^{\prime}$. Furthermore, it holds that $\theta^{*}\left(\omega^{\prime}\right)=\theta^{*}(\omega)$ and $\theta^{L}\left(\omega^{\prime}\right)=\theta^{L}(\omega)$, so the relative optimality gap is the same.

Finally, notice that $d_{\left(y^{L} \cup y^{*}\right)}\left(\omega^{\prime}\right) \equiv d_{(n, m)}\left(\omega^{\prime}\right)$ for $n, m \in$ $\{0, \ldots, k\}, y^{L} \subseteq \ell^{L}$ with $\left|y^{L}\right|=n$, and $y^{*} \subseteq \ell^{*}$ with $\left|y^{*}\right|=m$. This completes the proof. We capture this transformation by $\phi_{3}$ : $\phi_{2}\left(\phi_{1}(\Omega)\right) \rightarrow \phi_{2}\left(\phi_{1}(\Omega)\right)$.

We can simplify the derived expressions for $\omega \in \phi_{3}\left(\phi_{2}\left(\phi_{1}\right.\right.$ $(\Omega))$ ), since only the cardinality of the subset is important. The maxima are given by:

$$
\begin{aligned}
& \theta^{L}(\omega)=\sum_{n=1}^{k} \sum_{m=0}^{k}\left(\begin{array}{l}
k \\
n
\end{array}\right)\left(\begin{array}{l}
k \\
m
\end{array}\right) d_{(n, m)}(\omega), \\
& \theta^{*}(\omega)=\sum_{m=1}^{k} \sum_{n=0}^{k}\left(\begin{array}{l}
k \\
m
\end{array}\right)\left(\begin{array}{l}
k \\
n
\end{array}\right) d_{(n, m)}(\omega) .
\end{aligned}
$$

For all $R \in\{1, \ldots, k\}$, the global maximum constraints are:

$$
\sum_{r=1}^{R}\left(\begin{array}{l}
R \\
r
\end{array}\right) \sum_{n=0}^{k-R}\left(\begin{array}{c}
k-R \\
n
\end{array}\right)\left(d_{(n+r, 0)}(\omega)-d_{(n, r)}(\omega)\right) \leq 0 .
$$

Likewise, for all $R \in\{1, \ldots, \rho\}$ the $\rho$-Swap local maximum constraints are:

$$
\sum_{r=1}^{R}\left(\begin{array}{l}
R \\
r
\end{array}\right) \sum_{m=0}^{k-R}\left(\begin{array}{c}
k-R \\
m
\end{array}\right)\left(d_{(0, m+r)}(\omega)-d_{(r, m)}(\omega)\right) \leq 0 .
$$

\subsection{Step 4: simplify the structure}

The final transformation $\phi_{4}: \phi_{3}\left(\phi_{2}\left(\phi_{1}(\Omega)\right)\right) \rightarrow \phi_{3}\left(\phi_{2}\left(\phi_{1}\right.\right.$ $(\Omega))$ ) combines demand in a weighted manner. To be specific, the demand of the new instance $\omega^{\prime}$ is set to

$d_{(1,0)}\left(\omega^{\prime}\right)=\frac{1}{k} \sum_{n=1}^{k}\left(\begin{array}{l}k \\ n\end{array}\right) d_{(n, 0)}(\omega)$,

$d_{(1,1)}\left(\omega^{\prime}\right)=\frac{1}{k^{2}} \sum_{n=1}^{k} \sum_{m=1}^{k}\left(\begin{array}{l}k \\ n\end{array}\right)\left(\begin{array}{l}k \\ m\end{array}\right) d_{(n, m)}(\omega)$,

$d_{(0,1)}\left(\omega^{\prime}\right)=\frac{1}{k} \sum_{m=1}^{k}\left(\begin{array}{l}k \\ m\end{array}\right) d_{(0, m)}(\omega)$,

and zero otherwise. No other changes are performed. We prove in Lemma 6 that this transformation preserves global optimality of $x^{*}$ and $\rho$-Swap local optimality of $x^{L}$.

Lemma 6. Consider an MCP instance $\omega \in \phi_{3}\left(\phi_{2}\left(\phi_{1}(\Omega)\right)\right)$ where the global optimum $x^{*}(\omega)$ and $\rho$-Swap local optimum $x^{L}(\omega)$ differ at $2 k$ elements. There exists an MCP instance $\omega^{\prime} \in \phi_{3}\left(\phi_{2}\left(\phi_{1}(\Omega)\right)\right)$ such that $x^{*}(\omega)$ is still the global optimum and $x^{L}(\omega)$ still a $\rho$-Swap local optimum. The maxima satisfy the following relations:

$\theta^{L}\left(\omega^{\prime}\right)=k d_{(1,0)}\left(\omega^{\prime}\right)+k^{2} d_{(1,1)}\left(\omega^{\prime}\right)$,

$\theta^{*}\left(\omega^{\prime}\right)=k d_{(0,1)}\left(\omega^{\prime}\right)+k^{2} d_{(1,1)}\left(\omega^{\prime}\right)$,

with the necessary and sufficient constraints

$$
\begin{aligned}
d_{(0,1)}\left(\omega^{\prime}\right)-d_{(1,0)}\left(\omega^{\prime}\right) & \geq 0, \\
d_{(0,1)}\left(\omega^{\prime}\right)-d_{(1,0)}\left(\omega^{\prime}\right)-(k-\rho) d_{(1,1)}\left(\omega^{\prime}\right) & \leq 0, \\
d_{(0,1)}\left(\omega^{\prime}\right), d_{(1,0)}\left(\omega^{\prime}\right), d_{(1,1)}\left(\omega^{\prime}\right) & \in \mathbb{R}_{\geq 0} .
\end{aligned}
$$

Furthermore, the relative optimality gap of $\omega^{\prime}$ is equal to that of $\omega$.
Proof. Let $\omega \in \phi_{3}\left(\phi_{2}\left(\phi_{1}(\Omega)\right)\right)$ be an arbitrary instance where the global optimum and $\rho$-Swap local optimum differ at $2 k$ elements. Apply the final transformation to $\omega$ : $\omega^{\prime}=\phi_{4}(\omega)$. By substituting the defined demand weights, we see that the objective values of $x^{L}$ and $x^{*}$ are unaffected.

Since many $d_{(n, m)}\left(\omega^{\prime}\right)$ of the new instance are zero, the global maximum constraints (4) simplify by definition to:

$R\left(d_{(1,0)}\left(\omega^{\prime}\right)-d_{(0,1)}\left(\omega^{\prime}\right)-(k-R) d_{(1,1)}\left(\omega^{\prime}\right)\right) \leq 0$,

which must hold for all $R \in\{1, \ldots, k\}$. Notice that the constraint for $R=k$ is the most restricting, i.e., the global maximum constraints are satisfied if and only if $d_{(1,0)}\left(\omega^{\prime}\right)-d_{(0,1)}\left(\omega^{\prime}\right) \leq 0$. This constraint is indeed valid for $\omega^{\prime}$ :

$d_{(1,0)}\left(\omega^{\prime}\right)-d_{(0,1)}\left(\omega^{\prime}\right)=\frac{1}{k}\left(\theta^{L}(\omega)-\theta^{*}(\omega)\right) \leq 0$.

We conclude that $x^{*}$ is still a global optimum for $\omega^{\prime}$.

The final step is to check if the $\rho$-Swap constraints are valid for $x^{L}$ and $\omega^{\prime}$. Unfortunately, this part of the proof is somewhat cumbersome and is given in the Appendix. The corresponding constraint follows again from the defined demand weights, similarly to the global maximum constraints.

\subsection{Step 5: combining the results}

We can now prove Theorem 3 by using the four instance transformations and the three lemmas. In the proof we derive the maximum relative optimality gap of a $\rho$-Swap local maximum under the conditions stated in Theorem 3.

Proof of Theorem 3. Sequentially apply the instance transformations $\phi_{1}, \ldots, \phi_{4}$ to the original MCP instance $\omega$, resulting in a new instance $\omega^{\prime}$. We have shown that each transformation preserves the four mentioned properties. Hence, the transformation results in a family of worst-case instances with symmetric properties. This family can be described by the (abstract) relations from Lemma 6. Therefore, we are interested in the following optimisation model to determine the Swap locality gap. Maximise the relative optimality gap:

$\left(\theta^{*}-\theta^{L}\right) / \theta^{*}=\left(d_{(0,1)}-d_{(1,0)}\right) /\left(d_{(0,1)}+k d_{(1,1)}\right)$,

subject to

$$
\begin{aligned}
d_{(0,1)}-d_{(1,0)} & \geq 0, \\
d_{(0,1)}-d_{(1,0)}-(k-\rho) d_{(1,1)} & \leq 0, \\
d_{(0,1)}, d_{(1,0)}, d_{(1,1)} & \in \mathbb{R}_{\geq 0} .
\end{aligned}
$$

The maximum $(k-\rho) /(2 k-\rho)$ is attained by setting $d_{(0,1)}>0$, $d_{(1,0)}=0$, and $d_{(1,1)}=d_{(0,1)} /(k-\rho)$. See also Fig. 4 .

Although the proof of Theorem 3 describes how the worst-case MCP instances can be constructed, it is useful to give an explicit description. For $\rho \in \mathbb{N}_{\geq 1}$ and $k \in \mathbb{N}$ with $k \geq \rho+1$, the worst-case MCP instance has the following parameters. The number of opened bases $p$ is equal to $k$ and there are in total $|\ell|=2 p=2 k$ bases. The first $p$ bases correspond to a $\rho$-Swap Local Search optimum $\left(\ell^{L}=\{1, \ldots, p\}\right)$ and the last $p$ bases to the optimal solution $\left(\ell^{*}=\{p+1, \ldots, 2 p\}\right)$. There are $p(p+1)$ demand points, where the first $p^{2}$ points are covered by exactly one $\rho$-Swap base and one optimal base. These points have demand $(k-\rho)^{-1}$. The final $p$ demand points are covered by exactly one optimal base and have demand 1.

Our proof gradually builds a description of worst-case instances, which has the potential to be used for other combinatorial optimisation problems as well. In particular, we note that we reduce the number of demand parameters from $2^{|\ell|}$ to $(k+1)^{2}$ by symmetry, followed by another reduction to 3 demand parameters, and finally only one parameter remains. Since the worst-case instances are very symmetric, it would be interesting to see the effect on the optimality gap of small perturbations in the symmetry. 




(a) Instance $\omega \in \phi_{2}(\Omega)$. Artificial demand points are not shown.

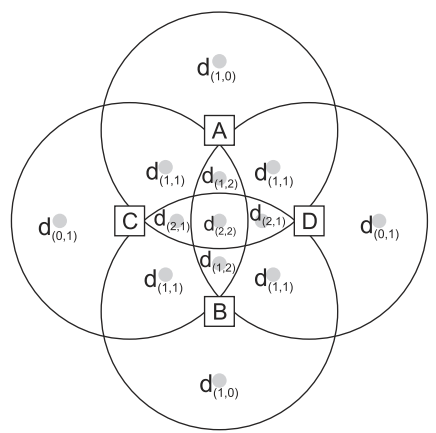

(b) New instance $\omega^{\prime}=\phi_{3}(\omega)$. Two points, $d_{(2,0)}$ and $d_{(0,2)}$, are not displayed.

Fig. 3. Third instance transformation $\phi_{3}$, assuming $\ell(\omega)=\{A, B, C, D\}, p(\omega)=2, \ell^{L}(\omega)=\{A, B\}$, and $\ell^{*}(\omega)=\{C, D\}$.

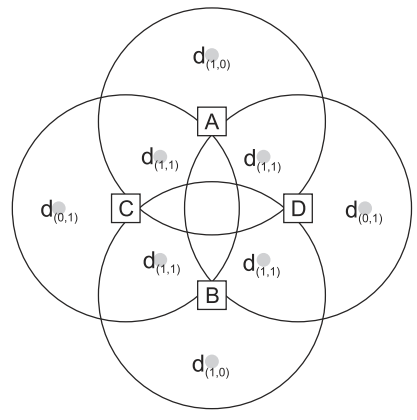

(a) Instance $\omega \in \phi_{4}(\Omega)$.

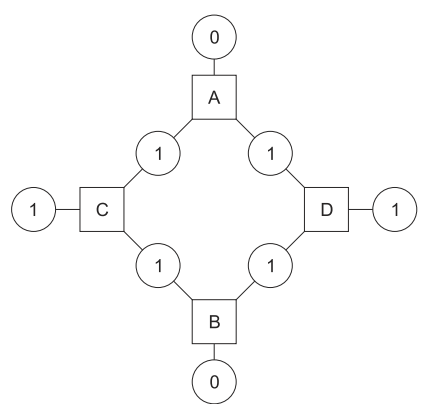

(b) Worst-case instance.

Fig. 4. Worst-case demand values, assuming $\ell(\omega)=\{A, B, C, D\}, p(\omega)=2, \ell^{L}(\omega)=\{A, B\}$, and $\ell^{*}(\omega)=\{C, D\}$.

\section{Acknowledgements}

This research is part of the REPRO research project on ambulance logistics, and is the result of the M.Sc. thesis of the first author. The REPRO project is a cooperation between CWI and the Delft University of Technology, financed by Technology Foundation STW under contract 11986. CWI is the national research institute for mathematics and computer science in The Netherlands.

\section{Appendix. Proof of swap local optimality}

We give the details to show that $\phi_{4}$ preserves the $\rho$-Swap local optimality of $x^{L}$, see also Lemma 6 . Let $R \in\{1, \ldots, \rho\}$ and recall the $\rho$-Swap constraint:

$$
\begin{aligned}
R\left(d_{(0,1)}\left(\omega^{\prime}\right)-d_{(1,0)}\left(\omega^{\prime}\right)-(k-R) d_{(1,1)}\left(\omega^{\prime}\right)\right) \\
=\frac{R}{k} \sum_{m=1}^{k}\left(\begin{array}{l}
k \\
m
\end{array}\right) d_{(0, m)}(\omega)-\frac{R}{k} \sum_{n=1}^{k}\left(\begin{array}{l}
k \\
n
\end{array}\right) d_{(n, 0)}(\omega) \\
\quad-\frac{R(k-R)}{k^{2}} \sum_{n=1}^{k} \sum_{m=1}^{k}\left(\begin{array}{l}
k \\
n
\end{array}\right)\left(\begin{array}{l}
k \\
m
\end{array}\right) d_{(n, m)}(\omega),
\end{aligned}
$$

which must be non-positive. First, we focus on the two negative terms:

$$
\begin{aligned}
- & \frac{R}{k} \sum_{n=1}^{k}\left(\begin{array}{l}
k \\
n
\end{array}\right) d_{(n, 0)}(\omega)-\frac{R(k-R)}{k^{2}} \sum_{n=1}^{k} \sum_{m=1}^{k}\left(\begin{array}{l}
k \\
n
\end{array}\right)\left(\begin{array}{l}
k \\
m
\end{array}\right) d_{(n, m)}(\omega) \\
\leq & -\frac{R}{k} \sum_{n=1}^{R}\left(\begin{array}{l}
k \\
n
\end{array}\right) d_{(n, 0)}(\omega) \\
& -\frac{R(k-R)}{k^{2}} \sum_{n=1}^{R} \sum_{m=1}^{k-R}\left(\begin{array}{l}
k \\
n
\end{array}\right)\left(\begin{array}{l}
k \\
m
\end{array}\right) d_{(n, m)}(\omega)
\end{aligned}
$$

$$
\begin{aligned}
& \leq-\sum_{n=1}^{R}\left(\begin{array}{l}
R \\
n
\end{array}\right) d_{(n, 0)}(\omega)-\sum_{n=1}^{R} \sum_{m=1}^{k-R}\left(\begin{array}{l}
R \\
n
\end{array}\right)\left(\begin{array}{c}
k-R \\
m
\end{array}\right) d_{(n, m)}(\omega) \\
& =-\sum_{r=1}^{R}\left(\begin{array}{l}
R \\
r
\end{array}\right) \sum_{m=0}^{k-R}\left(\begin{array}{c}
k-R \\
m
\end{array}\right) d_{(r, m)}(\omega) .
\end{aligned}
$$

Second, suppose we can bound the positive term of the $\rho$-Swap constraint as follows:

$\frac{R}{k} \sum_{m=1}^{k}\left(\begin{array}{l}k \\ m\end{array}\right) d_{(0, m)}(\omega) \leq \sum_{r=1}^{R}\left(\begin{array}{l}R \\ r\end{array}\right) \sum_{m=0}^{k-R}\left(\begin{array}{c}k-R \\ m\end{array}\right) d_{(0, m+r)}(\omega)$.

Combining (A.1) and (A.2) shows that the $\rho$-Swap constraints are valid for $\omega^{\prime}$, since constraints (5) hold for $\omega$.

Thus, only the proof of Eq. (A.2) remains. First apply an identity, also known as the Chu-Vandermonde identity:

$\frac{R}{k} \sum_{m=1}^{k}\left(\begin{array}{l}k \\ m\end{array}\right) d_{(0, m)}(\omega)=\frac{R}{k} \sum_{m=1}^{k} \sum_{r=0}^{m}\left(\begin{array}{l}R \\ r\end{array}\right)\left(\begin{array}{l}k-R \\ m-r\end{array}\right) d_{(0, m)}(\omega)$.

Next, we split the summation in (A.3) into two parts, corresponding to $r=0$ and $r>0$. Furthermore, we switch the order of summation and delete terms with binomial coefficients equal to zero. Consequently, (A.3) is equal to

$$
\begin{gathered}
\frac{R}{k} \sum_{m=1}^{k}\left(\begin{array}{c}
k-R \\
m
\end{array}\right) d_{(0, m)}(\omega)+\frac{R}{k} \sum_{r=1}^{k} \sum_{m=r}^{k}\left(\begin{array}{l}
R \\
r
\end{array}\right)\left(\begin{array}{c}
k-R \\
m-r
\end{array}\right) d_{(0, m)}(\omega) \\
=\frac{R}{k} \sum_{m=1}^{k-R}\left(\begin{array}{c}
k-R \\
m
\end{array}\right) d_{(0, m)}(\omega) \\
+\frac{R}{k} \sum_{r=1}^{R} \sum_{m=r}^{k-R+r}\left(\begin{array}{l}
R \\
r
\end{array}\right)\left(\begin{array}{l}
k-R \\
m-r
\end{array}\right) d_{(0, m)}(\omega)
\end{gathered}
$$




$$
\begin{aligned}
= & \frac{R}{k} \sum_{m=1}^{k-R}\left(\begin{array}{c}
k-R \\
m
\end{array}\right) d_{(0, m)}(\omega) \\
& +\left(1-\frac{k-R}{k}\right) \sum_{r=1}^{R} \sum_{m=0}^{k-R}\left(\begin{array}{c}
R \\
r
\end{array}\right)\left(\begin{array}{c}
k-R \\
m
\end{array}\right) d_{(0, m+r)}(\omega) .
\end{aligned}
$$

Notice that we have been able to obtain the right-hand side of Eq. (A.2), but there are some additional terms. Therefore, consider these additional terms:

$$
\begin{aligned}
& \frac{R}{k} \sum_{m=1}^{k-R}\left(\begin{array}{c}
k-R \\
m
\end{array}\right) d_{(0, m)}(\omega) \\
& \quad-\frac{k-R}{k} \sum_{r=1}^{R} \sum_{m=0}^{k-R}\left(\begin{array}{c}
R \\
r
\end{array}\right)\left(\begin{array}{c}
k-R \\
m
\end{array}\right) d_{(0, m+r)}(\omega) \\
& \leq \frac{R}{k} \sum_{m=1}^{k-R}\left(\begin{array}{c}
k-R \\
m
\end{array}\right) d_{(0, m)}(\omega)-\frac{k-R}{k} \sum_{m=1}^{k-R+1} R\left(\begin{array}{c}
k-R \\
m-1
\end{array}\right) d_{(0, m)}(\omega) \\
& \leq \frac{R}{k} \sum_{m=1}^{k-R}\left(\left(\begin{array}{c}
k-R \\
m
\end{array}\right)-(k-R)\left(\begin{array}{c}
k-R \\
m-1
\end{array}\right)\right) d_{(0, m)}(\omega) \leq 0 .
\end{aligned}
$$

As the sum of these extra terms is non-positive, we have shown that Eq. (A.2) is valid. Hence, the $\rho$-Swap optimality conditions hold for $x^{L}$ and $\omega^{\prime}$. Note that only the most restricting $\rho$-Swap local maximum constraints need to be included.

\section{References}

[1] R. Church, C. ReVelle, The maximal covering location problem, Pap. Reg. Sci. 32 (1) (1974) 101-108. http://dx.doi.org/10.1007/BF01942293.

[2] U. Feige, A threshold of $\ln n$ for approximating set cover, J. ACM 45 (4) (1998) 634-652. http://dx.doi.org/10.1145/285055.285059.

[3] Y. Filmus, J. Ward, The power of local search: maximum coverage over a matroid, in: 29th International Symposium on Theoretical Aspects of Computer Science, STACS 2012, 2012, pp. 601-612. http://dx.doi.org/10.4230/LIPIcs. STACS.2012.601.

[4] M.L. Fisher, G.L. Nemhauser, L.A. Wolsey, An analysis of approximations for maximizing submodular set functions-II, in: Polyhedral Combinatorics, Vol. 8, Springer, Berlin, Heidelberg, 1978, pp. 73-87. http://dx.doi.org/10.1007/ BFb0121195.

[5] R.B.O. Kerkkamp, Facility location models in emergency medical service: robustness and approximations (Master's thesis), Delft University of Technology, 2014, Available at repository.tudelft.nl.

[6] G.L. Nemhauser, L.A. Wolsey, M.L. Fisher, An analysis of approximations for maximizing submodular set functions-I, Math. Program. 14 (1)(1978) 265-294. http://dx.doi.org/10.1007/BF01588971. 